\title{
L'entrepreneuse et le temps : les femmes ont-elles besoin de dispositifs d'accompagnement spécifiques ?
}

\author{
Thierry Levy-Tadjine, Sibel Tokatlioglu et Jihene Zouiten \\ Université du Sud Toulon-Var
}

\section{Introduction}

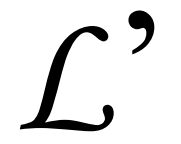

entrepreneuse est souvent reconnue pour être plus altruiste dans son aventure entrepreneuriale que son homologue masculin. Or, Levy-Tadjine, Defreyman et Paturel ${ }^{1}$ ont montré (dans le contexte français) que l'altruisme et sa conséquence, l'irruption d'acteurs plus nombreux dans le processus entrepreneurial, étaient générateurs de dissonances temporelles marquées entre l'entrepreneuse, son entourage et les parties prenantes de l'aventure entrepreneuriale (accompagnateurs, financeurs...).

Quelle est la conséquence de ce constat en termes d'accompagnement ? C'est à cette question que notre contribution ambitionne de répondre.

Pour ce faire, la singularité de l'entrepreneuriat féminin est d'abord discutée en regard d'études menées sur des contextes différents (français, turcs et américains). La généralisation de
L'éthos du profit ne constitue pas sa seule motivation à entreprendre. immigrés, et par le fait qu'elles associent une dimension affective et altruiste à leur aventure entrepreneuriale. En d'autres termes, l'éthos du profit ne constitue pas sa seule motivation à entreprendre. Comme l'a suggéré Mione ${ }^{4}$, elle se justifierait davantage par une logique d'effectivité et d'affectivité que par une logique d'efficacité ou d'efficience.

Il est également avéré que les femmes, lorsqu'elles entreprennent, sont plus hésitantes à s'endetter que les hommes et moins ambitieuses en termes de rentabilité ${ }^{5}$, comme le suggèrent l'étude de Carter $^{6}$ sur des femmes créatrices aux États-Unis ou celle de Ertubey ${ }^{7}$ sur les entrepreneuses turques. La faible propension à l'emprunt proviendrait en particulier des anticipations de discrimination au crédit que Levy-Tadjine ${ }^{8}$ qualifiait de discrimination perçue ${ }^{9}$ ainsi que de la taille souvent plus réduite des projets portés par les femmes. Ozar ainsi que Ertubey ${ }^{10}$ l'illustrent dans le cas des entrepreneuses turques en comparant leurs affaires à celles de leurs homologues masculins.

Ce résultat confirme imla singularité de l'entrepreneuriat féminin est ensuite relue en termes de dissonances temporelles. Cette relecture permet finalement de renouveler le débat sur l'accompagnement de l'entrepreneuriat féminin en offrant une justification à l'existence de dispositifs spécifiquement dédiés.

\section{La singularité apparemment universelle de l'entrepreneuriat féminin}

Comme l'ont suggéré Levy-Tadjine et Zouiten², l'entrepreneuriat féminin est souvent rapproché de l'entrepreneuriat ethnique et considéré comme singulier par le fait que les femmes entreprennent souvent par dépit ou par nécessité économique ${ }^{3}$, comme les plicitement l'hypothèse d'un entrepreneuriat par dépit, tout en suggérant la présence d'autres mobiles entrepreneuriaux que ceux usuellement avancés. Nous proposons de le relire dans les termes d'une dissonance temporelle marquée qui caractériserait l'entrepreneuriat féminin.

\section{Un rapport au temps singulier}

Pour Chalaye ${ }^{11}$, en effet, le rapport au temps singulier des femmes est encastré dans leur rôle social. L'auteur, reprenant des statistiques élaborées par l'Institut national de la statistique et des études économiques (INSEE) et par le programme EQUAL, rapporte qu'en 
France, les femmes consacrent deux fois plus de temps aux activités domestiques que les hommes (262 minutes/jour contre 140 pour les hommes d'après l'enquête emploi du temps de l'INSEE en 1999). Cet élément les rendrait plus polychroniques que les hommes, comme l'observait déjà Hall ainsi que Palmer et
Schoorman ${ }^{12}$. Mais la polychronicité qui constitue un des signes distinctifs de l'entrepreneur ${ }^{13}$ n'est pas la seule marque du rapport au temps singulier de l'entrepreneuse. Son orientation altruiste génère également des dissonances temporelles caractéristiques, comme l'illustre le cas Fak.

\section{Le Cas Fak}

Melle Fak, française d'origine marocaine, à première vue parfaitement intégrée mais ne trouvant pas d'emploi stable répondant à ses aspirations après des études en administration économique et sociale, décide de créer son affaire pour, explique-telle, « se réaliser socialement ». Son projet est un projet intégrationniste qui fait appel à son savoir-faire culturel tout en visant une clientèle autochtone de classe moyenne. Il s'agit de commercialiser des objets d'artisanat marocain à but décoratif en France. Par déficit de ressources financières, elle ne trouve pas de local en centre-ville et, contre l'avis de son conseiller, pressée d'entreprendre, elle signe un bail pour un local en Zone Franche Urbaine. Au final, elle ouvre son affaire qui se trouve en contradiction avec son « business model » initial... La clientèle qui fréquente sa boutique n'est pas celle qui était visée.

Paradoxalement, malgré son différend avec lui, elle regrette que le conseiller ne l'ait pas relancée par la suite, tout en niant la contradiction de son plan d'affaires alors qu'elle n'atteint pas le seuil de rentabilité. Lorsqu'on consulte l'analyse de l'accompagnateur, on se rend compte que celui-ci a un verdict très tranché sur l'affaire de Fak et qu'en tant que technicien, il considère que, dès qu'elle a « baissé les bras » et renoncé à chercher un local en centre-ville, « il n’y avait plus rien à faire...». Pour autant, en s'interrogeant sur ce constat d'échec de l'accompagnement, il finit après coup par déchiffrer l'empressement de Melle Fak. Si cette dernière s'est précipitée, c'est parce que l'une de ses motivations, alors cachée, à entreprendre consistait à « employer sa sœur», chômeuse en fin de droits, afin de lui redonner des droits. La sœur de Melle Fak, la portée du projet et l'objet réel de celui-ci, ont influencé directement le processus de création et, par la suite, son évaluation et la décision finale.

Ce cas traduit bel et bien un conflit de temporalité. Au temps (temps du projet) que préconisait le chargé de mission de la Boutique de Gestion, la porteuse de projet, pressée, a préféré un temps accéléré qui lui permettait de salarier sa sœur au plus vite. Son démarrage a davantage été motivé par l'objet altruiste que par le projet de l'aventure entrepreneuriale. Sa logique temporelle est donc entrée en conflit avec celle de la structure d'accompagnement

Dans ce cas, et du fait d'un écart d'éthos, l'orientation temporelle ${ }^{14}$ de l'entrepreneuse a différé du temps de l'accompagnateur qui cherchait à objectiver la temporalité du projet. Dans le cas Fak, cette dissonance temporelle a été accentuée par les temporalités d'autres acteurs gravitant autour de l'entrepreneur, en l'occurrence sa sœur. Or, comme le montrent LevyTadjine, Defreyman et Paturel (2005), cette multiplication des acteurs impliqués dans le processus entrepreneurial est la cause première des conflits de temporalité.

Les auteurs proposaient de résumer les conflits de temporalité au sein du processus entrepreneurial en distinguant trois grandes périodes au cours du processus entrepreneurial :

- Une zone de conflit de temporalité entre l'entrepreneur lui-même, d'une part, et les acteurs de son environnement de proximité - soit essentiellement les membres de sa famille -, d'autre part. La durée de ce conflit plus ou moins long durant le délai de réflexion d'accès à l'idée de créer, puis de passage de l'idée à l'intention de créer ou reprendre, pourra être influencée par des actions générales de sensibilisation à l'alternative que constitue l'entrepreneuriat par rapport au salarié. La formation, l'information avec ses nombreux dérivés ainsi que le rôle des récentes Maisons de l'entrepreneuriat interviennent sur cette zone afin de mettre un terme à et raccourcir la durée de ce conflit relativement personnel pour l'entrepreneur potentiel.

- Une zone de conflit de temporalité entre l'entrepreneur en herbe, décidé à se lancer dans les affaires et les acteurs de son environnement global et spécifique à son projet ${ }^{15}$. Ces derniers cherchent à obtenir le maximum de garantie avant d'assurer 
leur concours ou d'apporter leurs compétences dans tel ou tel domaine, ce qui a tendance à alourdir le temps de réalisation du projet par rapport aux souhaits de rapidité désormais dans l'esprit du créateur ou repreneur. Cette zone touche le lancement dans la conception du projet, la mise en forme du projet, les négociations diverses (avec les banquiers, les collectivités locales, le comité de pilotage de l'incubateur éventuel, le cédant sur le prix de la reprise, etc.) et de mise en œuvre effective de l'affaire (négociation de la présence du cédant pendant un temps dans l'affaire - quelle période ? Avec quelles attributions ? Négociation avec les organismes d'aide et d'accompagnement extérieurs, etc.).

- Une zone de conflit de temporalité entre l'entrepreneur et les acteurs désormais habituels de son environnement, quelle que soit la nature de ce dernier. Par exemple, la présence négociée au sein d'une pépinière posera le problème du terme de cette possibilité offerte par ce type de structure. De même, tous les acteurs normaux dans le démarrage de l'organisation seront en conflit quasi permanent avec le nouvel entrepreneur, les premiers étant dans une problématique de réduction de leurs risques pris auprès de leur nouveau client ou partenaire, avec ses conséquences en matière de temps, l'entrepreneur désirant de son côté réaliser au plus vite ses projets...

En conséquence, étant porteuses d'une plus forte dimension altruiste qui impliquerait davantage les portés ${ }^{16}$ du projet dans l'aventure entrepreneuriale, les femmes entrepreneuses rencontreraient davantage de conflits de temporalité semblables à celui décrit dans le cas Fak. Contre toute attente, et à l'opposé des conclusions de Levy-Tadjine et Zouiten (2005), ce résultat plaide alors pour la mise en place de réseaux d'accompagnement spécifiques aux femmes comme $A c$ tionElle ou PluriElle en France ou comme le Collectif des Femmes en Belgique au sein desquels les accompagnatrices, sensibilisées à la temporalité féminine, seraient plus performantes pour objectiver le temps du projet des entrepreneuses en herbe.

\section{Conclusion}

Notre recherche n'en n'est à ce stade qu'à sa phase exploratoire. Les nombreux travaux engagés sur l'en- trepreneuriat féminin, notamment au sein du Laboratoire ERMMES (Études et recherches méditerranéennes en management des entreprises) sur Toulon, devraient permettre l'approfondissement des pistes que nous avons esquissées dans cet article. En l'état, notre travail semble confirmer la pertinence d'une perspective centrée sur le rapport au temps de l'individu pour singulariser les phénomènes entrepreneuriaux. Sur le plan théorique, l'approfondissement de cette perspective pourrait mobiliser le courant théorique des sites symboliques d'appartenance ${ }^{17}$ qui permet également d'appréhender la variété des comportements sociaux à partir de l'éthos auquel les individus se rattachent.

\section{Les femmes entrepreneuses rencontreraient davantage de conflits de temporalité semblables à celui décrit dans le cas Fak.}

Sur le plan pratique, notre travail recommande d'encourager les réseaux de femmes entrepreneuses, ces dernières étant plus sensibles à une logique d'effectivité et d'affectivité qu'à une pure logique d'efficacité ou d'efficience qui prévaut dans les dispositifs d'accompagnement génériques usuels.

\section{Notes et références}

1 Levy-Tadjine T., J. De Freyman et R. Paturel (2005), « Les conflits de temporalité dans le processus entrepreneurial », communication à la $1^{\text {re }}$ conférence internationale COMICI, « Les valeurs du temps dans le développement des organisations », Brest, $1^{\mathrm{er}}$ décembre, cédérom.

2 Levy-Tadjine T. et J. Zouiten (2005), « Les femmes et les immigrés ont-ils besoin d'un accompagnement entrepreneurial spécifique ? », $\mathrm{IV}^{\mathrm{e}}$ congrès de l'Académie de l'entrepreneuriat, Paris 24-25 novembre, actes en ligne sur le site de l'Académie de l'entrepreneuriat.

3 Brush C.G. et R.D. Hisrich (1991), « Antecedent Influences on Women-owned Businesses », Journal of Managerial Psychology, vol. 6, $\mathrm{n}^{\circ}$ 2, p. 9-16.

4 Mione, A. (2004), «CIGALINE, entre affectivité, effectivité et efficacité. Bilan des cinq premières années de vie de l'unique Club d'investissement pour la gestion alternative de l'épargne solidaire au féminin », communication au $7^{\mathrm{e}}$ CIFPME, Montpellier, 27-29 octobre; Mione, A. (2005), « De l'affectivité à l'effectivité : l'évaluation par ses membres d'un réseau d'affiliation », communication présentée à l'atelier «Accompagnement des jeunes entreprises : entre 
darwinisme et assistanat» organisé par l'ERFI, le CEROM et l'AIREPME, Montpellier, 26 mai.

5 Kent, C.A., D.L. Sexton et K.H.Vesper (1983), Encyclopaedia of Entrepreneurship, Englewood Cliffs, N.J., Prentice Hall.

6 Carter, N.M. (2002), « The Role of Risk-orientation on Financing Expectations in New Venture Creation : Does Sex Matter? », Frontiers of Entrepreneurship Research, Babson College.

7 Ertubey N.O. (1993), Türkiye'de kadın girisimciligi: Mevcut durum, sorunlar ve öneriler. «Türkiye'de Kadin Girisimcilik» (N. Arat), p. 223-247. TES-AR Yayinlari, Ankara. (L'entrepreneuriat des femmes en Turquie : état actuel, problèmes et propositions, dans L'entrepreneuriat féminin en Turquie [édité par N. Arat], p. 223-247, Ankara, Éditions de TES-AR).

8 Levy-Tadjine, T. (2004), «L'entrepreneuriat immigré et son accompagnement en France ", thèse de doctorat (Ph.D. dissertation) en sciences de gestion, Université du Sud-Toulon-Var. Prix de la meilleure thèse en entrepreneuriat.

Consultable sur www.alexis.fr ou sur www.adreg.net.

9 L'auteur y montrait que cette perception altérait le modèle de la CSIP de Bruyat et qu'il importait peu que la discrimination soit réelle ou non. Le simple fait qu'elle soit anticipée atrophiait le projet et les aspirations du porteur (Bruyat, C. 1993, «Création d'entreprise : contributions épistémologiques et modélisation », thèse de doctorat ès sciences de gestion, Université Pierre-Mendez-France, Grenoble 2).

10 Ozar S. (2001) (Draft research report), « Barriers to Women's Micro and Small Enterprise Success in Turkey », International Policy Fellow, 2002 Center for Policy Studies, Central European University and Open Society Institute; Ertubey, N.O. (1993), op. cit.

11 Chalaye, M.N. (2005), «Faut-il féminiser le temps managérial ? Polychronicité, instantanéité et genre », communication à la $1^{\text {re }}$ conférence internationale COM-ICI, « Les valeurs du temps dans le développement des organisations », Brest, $1^{\text {er }}$ décembre, cédérom.
12 Hall, E.T. (1983), « The Dance of Life : The Other Dimension of Time », cité dans Chalaye (2005), op. cit.; Palmer D.K. et D.F. Schoorman (1999), « Unpackaging the Multiple Aspects of Time in Polychronicity », Journal of Managerial Psychology, vol. 14.

13 Kaish S. et B. Gilad (1991), « Characteristics of Opportunity Search for Entrepreneurs versus Executives : Sources, Interests, General Alertness », Journal of Business Venturing, vol. $6, \mathrm{n}^{\circ} 1$, p. 45-61. En interviewant 51 entrepreneurs et 36 cadres d'un grand établissement financier et en comparant leurs réponses, les auteurs ont souligné que la vigilance entrepreneuriale que les entrepreneurs cultiveraient les conduiraient, en effet, à ne pas segmenter temps professionnel et temps personnel. Comme le notent Chabaud et Ngijol, la vigilance des premiers «s'exerce dans des lieux plus inhabituels : à la différence du manager, l'entrepreneur sera vigilant en dehors de son temps et de son lieu de travail habituels. En fait, la vigilance s'apparente avant tout à un état d'esprit de l'entrepreneur qui l'incite finalement à ramener tout ce qu'il voit ou entend à son affaire, et cela, de manière continue, sans qu'il n'existe de cloisonnement strict entre vie professionnelle et vie privée » (Chabaud et Ngijol 2005, « La contribution de la théorie des réseaux sociaux à la reconnaissance des opportunités de marché », Revue internationale PME, vol. 18, $\mathrm{n}^{\circ} 1$ p. 34).

14

Chebat J.C. et V. Venkatesan (1991), Time and Consumer Behaviour, Montréal, UQAM.

15 Paturel, R. (1997), Pratique du management stratégique, Grenoble, Presses Universitaires de Grenoble, 150 p.

16 Pour Levy-Tadjine (2004), les portés désignent tout à la fois le projet lui-même et les partenaires qui en sont tributaires, sans avoir la possibilité d'en influencer directement la réalisation immédiate. On peut ainsi penser à de futurs collaborateurs du porteur de projet que celui-ci a prérecrutés ou à son entourage. Ils adhèrent au projet et n'attendent que sa réalisation. Même indirecte, leur influence n'en n'est pas moins importante, et ils contribuent à l'évaluation du projet, d'autant que, comme l'illustre le cas Fak, ils sont souvent porteurs d'une temporalité différente de celle qui anime l'entrepreneur.

17 Zaoual, H. (1992), «La méthodologie des sites symboliques », Cultures et Développement, $\mathrm{n}^{\circ} 10 / 11$, septembre. 